AAIA-99-1954

\title{
A MODE DETECTION METHOD USING THE AZIMUTHAL DIRECTIVITY OF A TURBOFAN MODEL
}

\author{
R. H. Thomas \\ AIAA Senior Member, Research Associate Professor \\ Mechanical Engineering Department, Virginia Tech \\ Virginia Consortium (VCES), 303 Butler Farm Road, Suite 101, Hampton, Virginia 23666 \\ F. Farassat \\ AIAA Associate Fellow, Sr. Research Scientist \\ Aeroacoustics Branch, NASA Langley Research Center \\ Hampton, Virginia 23681-2199 \\ L. R. Clark \\ Aerospace Engineer \\ Aeroacoustics Branch, NASA Langley Research Center \\ Hampton, Virginia 23681-2199 \\ C. H. Gerhold \\ AIAA Member, Sr. Research Engineer \\ Aeroacoustics Branch, NASA Langley Research Center \\ Hampton, Virginia 23681 \\ J. J. Kelly \\ AIAA Senior Member, Aeronautical Engineer \\ Lockheed Martin Engineering and Sciences Company \\ Hampton, Virginia 23681-2199 \\ L. E. Becker \\ Aeronautical Engineer \\ Lockheed Martin Engineering and Sciences Company \\ Hampton, Virginia 23681-2199
}

\begin{abstract}
$\underline{\text { Abstract }}$
The azimuthal, far field directivity of a scale fan model was measured in high resolution. The model is a 12 inch diameter rotor with 16 blades followed by 40 stator vanes. The tests were conducted at the nominal $100 \%$ speed corresponding to a tip speed of $905 \mathrm{ft} / \mathrm{sec}$. Measurement of the radiated sound field, forward of the fan, was made in an anechoic chamber

Copyright (C) 1999 by the American Institute of Aeronautics and Astronautics, Inc. No copyright is asserted in the United States under Title 17, U.S. Code. The U.S. Government has a royalty-free license to exercise all rights under the copyright claimed herein for Governmental Purposes. All other rights are reserved by the copyright owner.
\end{abstract}

with an inflow control device and a baffle separating the aft and forward radiated interaction noise. The acoustic field was surveyed with a circular hoop array of 16 microphones which was moved to 14 axial stations. At each axial station the hoop was rotated in half-degree increments to take 736 points in the azimuthal angle. In addition to sound pressure level, the phase angle relative to a reference microphone was measured at each point. The sound pressure level is shown to vary in patterns by $10-15 \mathrm{~dB}$ especially for the fundamental tone but also for the first and second harmonic. A far field mode detection method has been developed and used with the data which determines the modes generated by the fan and which then interact to form the azimuthal directivity. 


\section{Nomenclature}

BPF blade passage frequency

2BPF first harmonic

3BPF second harmonic

D fan rotor diameter, $1.0 \mathrm{ft}$

dB decibel, ref $20 \times 10^{-5} \mathrm{~N} / \mathrm{m}^{2}$

ICD inflow control device

SPL sound pressure level, $\mathrm{dB}$

$\mathrm{x}$ axial coordinate

$\psi \quad$ polar angle relative to engine axis

$\theta \quad$ azimuthal angle around engine axis

\section{$\underline{\text { Introduction }}$}

Far field acoustic testing of turbofan engines has traditionally been done with the assumption of axisymmetry of the radiated sound field about the axis of the engine. That is, measurement of the polar angle was done at a single azimuthal angle, typically directly to one side of the engine. When reviewing typical engine or experimental fan tests, it appears that this symmetry about the engine axis is rarely verified.

However, in a few cases azimuthal directivities were documented. For the similar case of counter-rotating propellers, a helical pattern was observed in the far field. ${ }^{1}$ A theory was developed for the acoustic interference pattern of propellers of equal blade number and rotational speed. ${ }^{2}$ In another case, an investigation of hybrid inlets was done with measurements made in two azimuthal angles around a JT15D engine and did show considerable variation but was not investigated further. ${ }^{3}$

More recently, the authors tested the same 12 inch fan model used in this investigation. The fan was tested in a wind tunnel and large azimuthal variations in the far field of the fan were measured by 15 sideline microphones, each at a different azimuthal angle. ${ }^{4}$ These variations in the BPF tone, up to $20 \mathrm{~dB}$, were attributed to standing wave patterns in the far field caused by the interaction of counter-rotating modes. These counter-rotating modes were attributed to rotorstator interaction modes interfering with extraneous modes produced by an interaction between the rotor and a non-uniform rub strip.

However, at the $2 \mathrm{BPF}$ tone where large variations were also present, it was not clear how the extraneous modes could propagate in order to create standing wave interaction with the single propagating mode due to rotor-stator interaction at the $2 \mathrm{BPF}$ frequency.

Ganz published very clear results of azimuthal directivity measurements on a different 12 inch fan model tested with an inflow control device. ${ }^{5}$ In this study, cylindrical rods were placed upstream of the rotor to create an inflow-rotor interaction. Measurements were taken in the far field in both polar and azimuthal angles but only for the BPF tone which was cut-off for the rotor-stator interaction cut-on for the rod wake-rotor interaction.

Ganz showed that large azimuthal variation in the BPF tone level occurred when two spinning modes were propagating. The measurements also clearly showed that the annular period of the azimuthal variations was $2 \pi\left(m_{1}-m_{2}\right)$ degrees, where $\mathrm{m}_{1}$ and $\mathrm{m}_{2}$ are two spinning mode orders. Ganz concluded that the large azimuthal variations would not be present if tones were dominated by only one spinning order.

However, it would seem that in practical applications that there would exist multiple modes together with extraneous modes from a variety of sources. This situation would seem to make the presence of azimuthal patterns a strong possibility.

This motivated an effort to better understand the identification of the sources and propagation of the modes which produce the azimuthal directivities. This was done by the authors who reported work where a circular array of microphones was used to survey the far field of the same 12 inch fan used in this work ${ }^{6}$.

The circular array of microphones was used to survey the azimuthal variations. The fundamental, first, and second harmonic tones were found to vary by 10 $15 \mathrm{~dB}$ in the azimuthal angle. The second harmonic tone was found to have a clearly defined 40 lobe pattern. A mode detection method was partially developed and used to identify the $m=-24,-15,-6$, and 3 circumferential modes.

This method was considered attractive because it uses only far field data and therefore, in contrast to other methods, does not require intrusive measurements or large numbers of microphones installed in the inlet. A circular array of microphones in the far field would not only give more accurate measurements of the far field including azimuthal variations, but in addition, could be used to identify the modes which radiate to the far field and produce those patterns. Therefore, the continued development of this promising experimental and data analysis method is the subject of this paper.

\section{Fan Model}

The fan model used for these experiments was a 12 inch diameter fan which was scaled from the Pratt and Whitney 17 Inch Advanced Ducted Propeller model. The fan model was originally constructed in 1993 and details of the design and description of its features have been previously reported. ${ }^{4,7}$ Only relevant features will be described here.

The fan has 16 wide chord rotor blades with a hub-to-tip ratio of 0.445 . In scaling from the 17 inch to the 12 inch model tip speed was held constant, therefore 
the $100 \%$ design speed at standard atmospheric conditions is $17188 \mathrm{rpm}$ yielding a tip speed of 905 $\mathrm{ft} / \mathrm{sec}$. The blade angle setting is at the takeoff condition and the fan pressure ratio is 1.27 at the $100 \%$ speed.

Although the fan was capable of interchangeable stator vane sets the experiment reported here was done with a 40 vane stator set with a rotor-tostator spacing of 2.0 based on the rotor mid-span chord. This stator vane set is a cut-off design for the BPF tone.

The rub strip has been identified in the past as a potential interaction source due to nonuniformity in the tip clearance. ${ }^{4}$ However, in this experiment care was taken to insure that the tip clearance was uniform. The rub strip used in this experiment had a static tip clearance of 0.030 inches with a maximum circumferential variation of 0.002 inches. Running tip clearance values were not taken. In addition, the rub strip was constructed in a way such that there were no joints or discontinuities in either the circumferential or axial direction in the part of the rub strip that was closest to the rotor.

The fan cowl was a flight type cowl whose internal contour was scaled from the Pratt and Whitney 17 inch model. The stator assembly provides the only structural support for the cowl. An ICD was attached to the fan cowl on the exterior of the inlet. The purpose of the ICD was to control inflow turbulence in order to allow proper simulation of flight type noise characteristics in a static ground test facility that was used in this work. The ICD was modeled after the NASA Lewis design for a compact ICD. ${ }^{8}$ A schematic of the fan and ICD installation is shown in Figure 1.

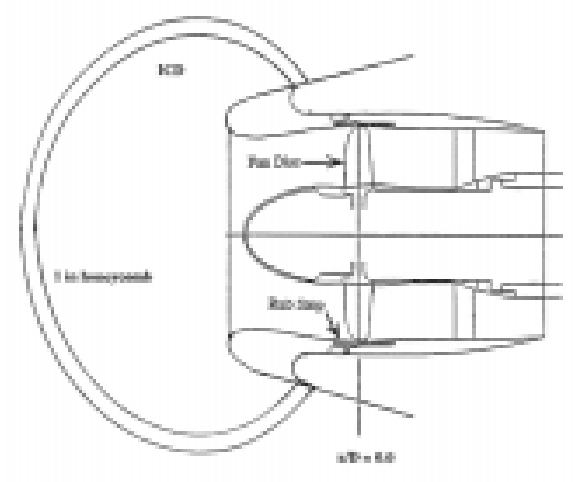

Figure 1. 12 inch fan with installation of the inflow control device.

\section{Facility and Model Arrangement}

The experiment was conducted in the Anechoic Noise Research Facility of NASA Langley Research Center. The internal dimensions of the test area (inside wedge tips ) was $27.5 \mathrm{ft}$ by $24 \mathrm{ft}$. The walls are covered with wedges which are 3 feet thick and yield an absorption coefficient greater than 99 percent above a frequency of $100 \mathrm{~Hz}$. The 12 inch model was mounted on a truss structure in the corner of the facility in order to direct the turbine exhaust out of the anechoic chamber. Air was allowed in the chamber through an air inductor system in the roof of the chamber.

A possible contributor to the azimuthal directivities that has been raised before is the interaction of the aft radiated noise with the forward radiated fan noise. To minimize this interaction source, a large baffle wall was constructed so that only the fan inlet protruded into the anechoic chamber where the far field measurements were taken. The wall was 8 feet square and covered on both sides with 3 inch thick acoustic foam. The location of the baffle relative to the model is shown in Figure 2 and it is also seen in the photograph in Figure 3.

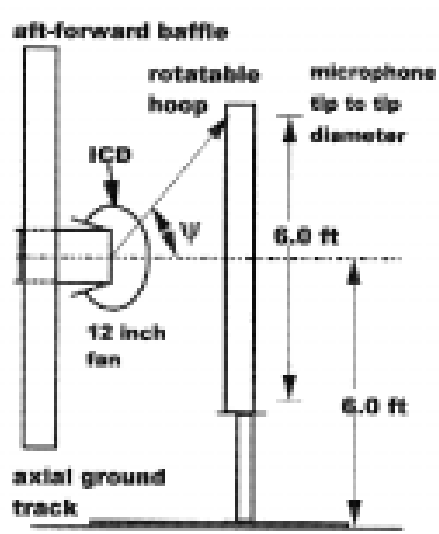

a) side viow

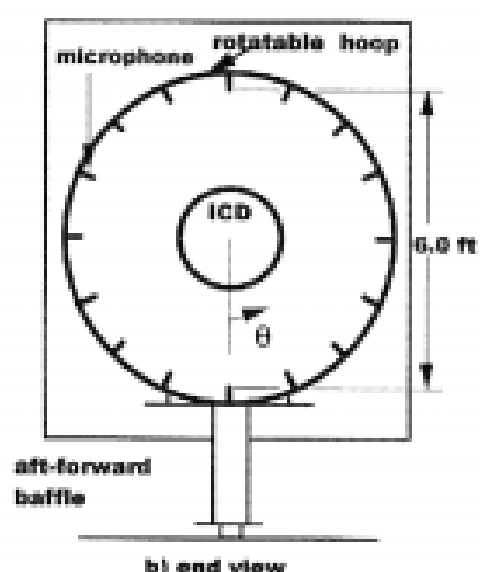

Figure 2. Circular microphone array and orientation relative to the 12 inch fan, a.) side view, b.) end view. 


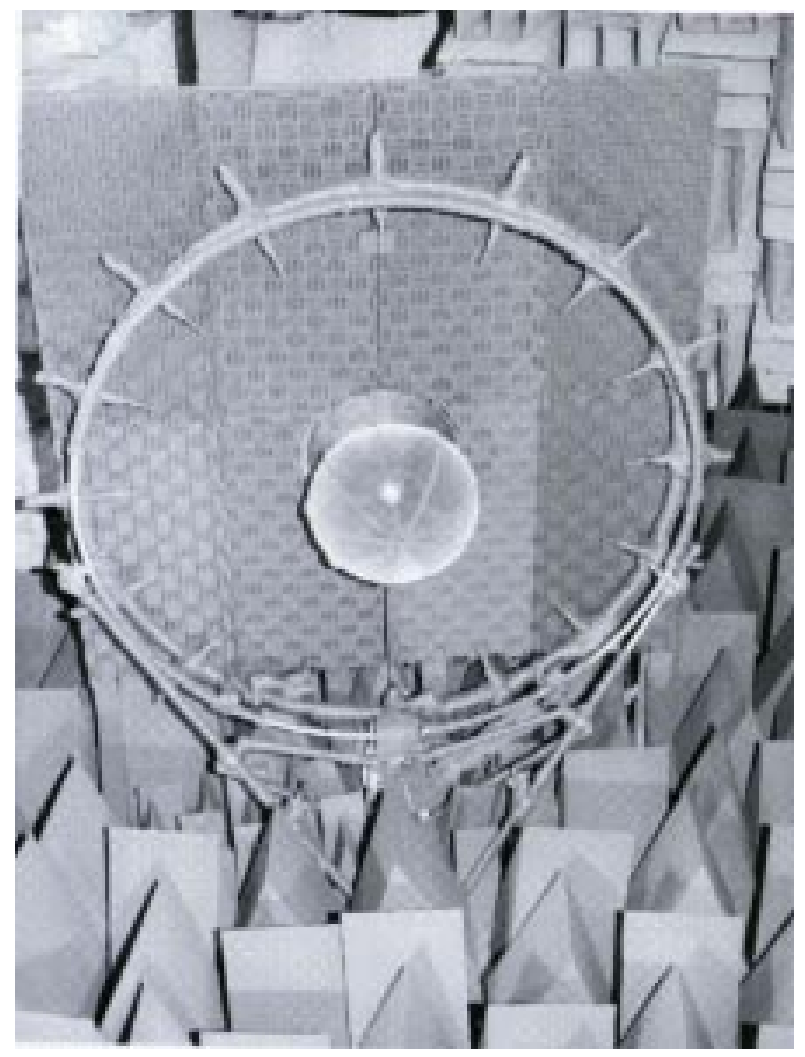

Figure 3. Photogaph of the anechoic chamber, circular microphone array, baffle wall, and 12 inch fan with ICD, all in run configuration.

A microphone traverse system was part of the facility that allowed for axial and azimuthal traverses. A circular hoop was mounted on a sled that could move in the axial direction on a ground track. The axial traverse could move the sled in the axial direction from $x / D=$ 0.0 to $x / D=15$ in front of the fan. The $x / D=0.0$ station was at the fan rotor stacking axis. Once at an axial location, the hoop could be rotated in the azimuthal direction.

Installed on the hoop are 16 microphones evenly spaced for an angular separation of 22.5 degrees. This allows for complete coverage in the azimuthal direction in high resolution. Both the axial and azimuthal traverses are driven by stepper motors with accuracy of more than $1 / 100^{\text {th }}$ of an inch. A schematic of the traverse system relative to the model is shown in Figure 2. Installed on the hoop, the 16 microphone diaphrams were on a 6 foot diameter circle or 6 fan diameters.

The standard acoustic foam wedges, 3 feet in height, were installed around the axial traverse as much as possible after the traverse was moved to each axial station. In addition, the hoop itself was covered in foam with a thickness of about 1 inch. Therefore, the presence of the traverse and hoop structure had a minimal impact on the performance of the anechoic chamber.

\section{Description of Experiment}

The experiment consisted of an extensive measurement of the radiated sound field of the 12 inch fan at one operating condition. The corrected speed of the fan was set at $17188 \mathrm{rpm}$, corresponding to the 100 $\%$ speed condition and corrected for pressure and temperature. The speed of the fan was held constant within a maximum of $\pm 40 \mathrm{rpm}$, most of the time the variation was typically $25 \mathrm{rpm}$. The array of microphones was positioned at axial stations exactly 9 inches apart, 3/4 of a fan diameter, starting at the $x / D=$ 0.0 station aligned with the fan rotor stacking axis. The $x / D=0.0$ station would correspond to a polar angle, $\psi$, of 90 degrees and station 14 at $x / D=9.75$ would correspond to $\psi=17.1^{\circ}$.

At each axial station the circular hoop was rotated in increments of 0.5 degrees for a total of 46 azimuthal stations for a total of 736 points. Therefore, with 14 axial stations, the total number of far field points taken was 10,304. Each axial station would require about 3 hours of continuous test time to acquire the data. This time included settling time after each rotation before data were taken, but much of the time was taken by storage time before the transient data recorder was ready to acquire the next set of data.

\section{$\underline{\text { Instrumentation and Data Analysis }}$}

The acoustic sensors used were 16 Bruel and Kjaer 4136 1/4 inch microphones mounted on the circular hoop. The microphone signals were sampled at $62.5 \mathrm{kHz}$ and low pass filtered at $30 \mathrm{kHz}$ and high pass filtered at $300 \mathrm{~Hz}$. At each measurement location the 16 microphones were sampled simultaneously. After a separate study the acquisition time was set at 4 seconds. A Hanning (cosine tapered) window was applied to each time series block. Microphone calibration corrections, including the frequency response, were applied to the signals. Then the sound pressure level spectra were computed from 30 averages of fast Fourier transforms each of 8192 points. The spectra were computed up to $15 \mathrm{kHz}$, which was enough to include the $3 \mathrm{BPF}$ tone. The peak level of the BPF, 2BPF, and $3 \mathrm{BPF}$ tones were identified from each spectra in order to be plotted. These tones could be identified exactly using a 16 per revolution signal from a shaft mounted sensor that was recorded together with the microphone signals.

The mode detection method that was used in this work has been extended beyond the preliminary use of this method in Reference 6 . The extension includes 
the identification of both circumferential and radial modes. This extension, however, requires the sound pressure level, as before, and the phase of the acoustic signal in the far field. This required that the data acquisition system be calibrated for both amplitude and phase.

The frequency response function (FRF) of the acquisition system can provide both channel phase and gain corrections due to the system response. A $1 / 2$ inch microphone was driven in reverse as a noise source and was coupled, with a specially made housing, to each of the $1 / 4$ inch acquisition microphones. The $1 / 2$ inch source microphone was driven with a white noise signal that was recorded on the $17^{\text {th }}$ channel as the reference for the system calibration. The usual method of computing the FRF, H(f), was then employed between each microphone and the reference signal:

$$
H(\mathrm{f})=\frac{\mathrm{G}_{\mathrm{io}}(\mathrm{f})}{\mathrm{G}_{\mathrm{ii}}(\mathrm{f})}
$$

where

$$
\begin{aligned}
& \mathrm{G}=\text { auto/cross-spectrum } \\
& \mathrm{i}=\text { input signal } \\
& \mathrm{O}=\text { output signal }
\end{aligned}
$$

Thus, the calibrated phase, $\phi_{c}$, of each channel, $\mathrm{n}$, is given by

$$
\phi_{c n}(\mathrm{f})=\tan ^{-1}\left\{\frac{\operatorname{Im}\left[H_{n}(\mathrm{f})\right]}{\operatorname{Re}\left[\mathrm{H}_{\mathrm{n}}(\mathrm{f})\right]}\right\}
$$

and the gain by $\left|H_{n}\right|$. Next, the phase differences were computed between each channel and channel 1 which was designated as the reference microphone for the data runs:

$$
\Delta_{c} \phi_{n}(\mathrm{f})=\phi_{\mathrm{cn}}(\mathrm{f}) \pm \phi_{\mathrm{cl}}(\mathrm{f})
$$

These calibrated phase differences must be subtracted from the measured acoustic signals obtained from the data runs. The gain values were used as corrections to obtain the amplitudes of the acoustic signals. A corrected sound-pressure level (SPL) was produced which used the system frequency response,

$$
\operatorname{SPL}(f)=\operatorname{SPL}_{M}(f) \pm 20 \log \left|\frac{\mathrm{H}(\mathrm{f})}{\mathrm{H}(1 \mathrm{kHz})}\right|
$$

Here $\operatorname{SPL}_{M}(\mathrm{f})$ was the measured signal and the correction includes a frequency dependent amplitude term that is, in turn, corrected relative to the $1 \mathrm{kHz}$ level obtained with a standard microphone calibrator. Therefore, each microphone's measured amplitude was corrected as a function of frequency. These frequency dependent corrections were as much as $2-3 \mathrm{~dB}$ over the $15 \mathrm{kHz}$ range.

\section{Complex Amplitude Computation}

The pressure-time history from each channel was segmented for the ensemble averaging that was performed during the computation of the auto/cross spectra. This technique was more effective at removing uncorrelated noise than other averaging methods. Both the calibration and the actual model data were ensemble averaged using the following expression ${ }^{9}$ in digital form

$$
G_{m n}(f)=\frac{2}{n_{d} N \Delta t} \sum_{j=1}^{n_{d}} P_{m j}^{*}(f) P_{n j}(f)
$$

where

$\mathrm{m}, \mathrm{n}=$ channel indices

$\mathrm{P}=$ fast Fourier transform (FFT)

$\mathrm{N}=$ number of points in FFT

$\mathrm{j}=$ record index (for ensemble averaging)

$\Delta \mathrm{t}=$ reciprocal of the sample rate

$\mathrm{n}_{\mathrm{d}}=$ number of records

For the model data, the channel phase differences are given by

$$
\Delta_{\mathrm{d}} \phi_{\mathrm{n}}(\mathrm{f})=\tan ^{-1}\left\{\frac{\operatorname{Im}\left[\mathrm{G}_{\mathrm{ln}}(\mathrm{f})\right]}{\operatorname{Re}\left[\mathrm{G}_{1 \mathrm{n}}(\mathrm{f})\right]}\right\}
$$

For each frequency bin, the system phase differences are subtracted from the test values to determine acoustic phase:

$$
\Delta \phi(\mathrm{f})=\Delta_{\mathrm{d}} \phi_{\mathrm{n}}(\mathrm{f}) \pm \Delta_{\mathrm{c}} \phi_{\mathrm{n}}(\mathrm{f})
$$

The channel phase angles are then set by the following procedure

$$
\Delta \phi_{1}(\mathrm{f})=0
$$

Sound-pressure levels which were computed for each frequency bin in the signal processing software were used to calculate the magnitude of the complex pressure amplitude. In the software, SPL was determined by way of the auto spectrum (see Eq. (5)) through the following steps for the mean-square acoustic pressure, 


$$
\left\langle p^{2}(f)\right\rangle=\frac{G_{n n}(f)}{N \Delta t}
$$

the sound pressure level,

$$
\operatorname{SPL}(\mathrm{f})=10 \log \left[\frac{\left\langle\mathrm{p}^{2}(\mathrm{f})\right\rangle}{\mathrm{p}_{\text {ref }}^{2}}\right]
$$

and then the complex amplitude can be expressed as

$$
|\mathrm{p}|=\sqrt{2\left\langle\mathrm{p}^{2}\right\rangle}=\sqrt{2} \mathrm{P}_{\text {ref }} 10^{\frac{\mathrm{SPL}}{20}}
$$

Thus, an ensemble-averaged value for $|\mathbf{p}|$ is produced. Finally, in terms of real and imaginary parts, the computed complex pressure amplitude is given as

$$
\mathrm{p}(\mathrm{f})=\mid \mathrm{p}(\mathrm{f})\left[\cos \Delta \phi_{\mathrm{n}}(\mathrm{f})+\mathrm{i} \sin \Delta \phi_{\mathrm{n}}(\mathrm{f})\right]
$$

Included would be any system phase value that was calibrated by referencing to microphone number 1 at its first rotation position at each given axial station.

\section{Error and Repeatability Analysis}

The objective of this test required a significant effort to minimize possible sources of error and several tests to quantify the repeatability of the results.

One major source of possible error was in simply positioning the microphones in the far field. The experiment was automated and required the circular array to move to 14 stations in the axial direction and to 46 stations in the azimuthal direction.

The circular array itself was a large flexible structure. A laser was mounted on a two axis leveling device to position the linear track and insure that the center of the circular array would stay on the line of the axis of the fan as it was moved from station to station. This laser was removed once the installation was complete and a second laser was permanently mounted on the far wall and aimed at the baffle surrounding the fan. The wall laser was positioned so that it would aim along a line exactly three feet above the axis of the fan that hit a target point on a small plate mounted on the baffle. In this way, as the array moved to each axial station, the laser could be used to check alignment and adjustments could be made using two outriggers on the array. The small plate and the two outriggers are visible in Figure 3.

These factors resulted in an accuracy of $3 / 8$ inch in positioning a given microphone anywhere in the measurement space. However, once the array was at a given axial location the stepper motor could rotate the array with an accuracy of $1 / 100^{\text {th }}$ of an inch and the axial stepper motor had the same accuracy in axial position. Therefore, while the absolute error in location was $3 / 8$ inch, maximum, the error in spacing, rotational or axial, is $1 / 100^{\text {th }}$ of an inch.

A set of five experiments was performed to specifically measure repeatability. The hoop was not rotated for this set. The hoop was stationed at the two extremes of the axial traverse and five samples were taken at each location. The acquisition window length for each was 8 seconds. Then the 8 -second sample was partitioned into a 2-second sample, a 4-second sample and an 8-second sample. In this way information could be provided to, at least partially, answer two key questions. First, the mean and standard deviation could be calculated for the five repeats. And second, the effect of ensemble averaging could be determined.

The results obtained at Station 17, most distant from the fan, were only slightly different from the results at Station 1, therefore, the results at Station 1 are presented in Table 1. The mean error for sound pressure level is seen as typical at about $1 \mathrm{~dB}$. Without much available reference for measurements of phase, it is difficult to assess phase stationarity. However, considering the experiment it would seem that this would be considered good repeatability of the phase angle. The increased sampling window length is seen to increase the repeatability considerably for the phase and

\begin{tabular}{|c|c|c|c|c|}
\hline & $\begin{array}{c}\text { Sampling } \\
\text { time }\end{array}$ & $2 \mathrm{Sec}$. & $4 \mathrm{Sec}$. & $8 \mathrm{Sec}$. \\
\hline \multirow[t]{2}{*}{ SPL } & Mean & $1.3 \mathrm{~dB}$ & $1.1 \mathrm{~dB}$ & $0.8 \mathrm{~dB}$ \\
\hline & $\begin{array}{l}\text { Standard } \\
\text { Deviation }\end{array}$ & $1.3 \mathrm{~dB}$ & $1.1 \mathrm{~dB}$ & $0.8 \mathrm{~dB}$ \\
\hline \multirow{2}{*}{$\begin{array}{l}\text { Phase } \\
\text { Angle }\end{array}$} & Mean & $17 \mathrm{deg}$. & $15 \mathrm{deg}$. & $9 \mathrm{deg}$. \\
\hline & $\begin{array}{l}\text { Standard } \\
\text { Deviation }\end{array}$ & $23 \mathrm{deg}$. & $18 \mathrm{deg}$. & $14 \mathrm{deg}$. \\
\hline
\end{tabular}
to a lesser extent for the sound pressure level.

Table 1. Repeatability Statistics Using Five Data Sets for the BPF Tone as a Function of Acquisition Window.

In a compromise of test time, storage space and other resources an acquisition window length of 4 seconds was determined for the experiment based on these results. However, a preliminary diagnostic experiment had been done with a 2 -second acquisition window length although not all of the system and microphone corrections were applied to this data. Therefore, the experiment with the 2 -second window is not considered an exact repeat experiment for comparison to the higher quality experiment with the 4second window.

\section{Experimental Results}

The acoustic data collected were reduced to the three tone levels, BPF, $2 \mathrm{BPF}$, and $3 \mathrm{BPF}$ as a 
function of azimuthal angle and axial station. At the $100 \%$ speed, at standard conditions, the BPF would be $4583 \mathrm{~Hz}$. The azimuthal variation of all three tones will be shown at two of the 14 axial stations to demonstrate the detail of the measurements followed by contour plots that show the entire far field.

The first axial station for which data are presented corresponds to the polar angle of $\psi=90^{\circ}$. Figure 4a plots the BPF tone as a function of azimuthal angle for the data taken with the 4-second window. The high resolution measurements do indeed show patterns that were well defined with amplitudes of up to $15 \mathrm{~dB}$. Figure $4 \mathrm{~b}$ shows the BPF tone for the preliminary experiment done with 2 -second data. For reasons described above, the two experiments are not expected to repeat in amplitude. However, the comparison of Figures $4 \mathrm{a}$ and $4 \mathrm{~b}$ do show the remarkable repeatability of the azimuthal patterns even though they were taken one month apart. Figure $4 \mathrm{~b}$ is the only data from the preliminary experiment to be shown, all other data are from the experiment with the 4-second window.

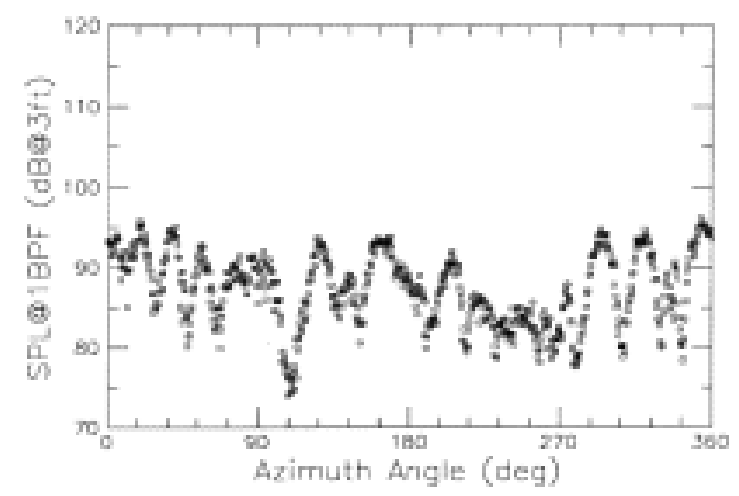

a.) BPF data with 4-second data.

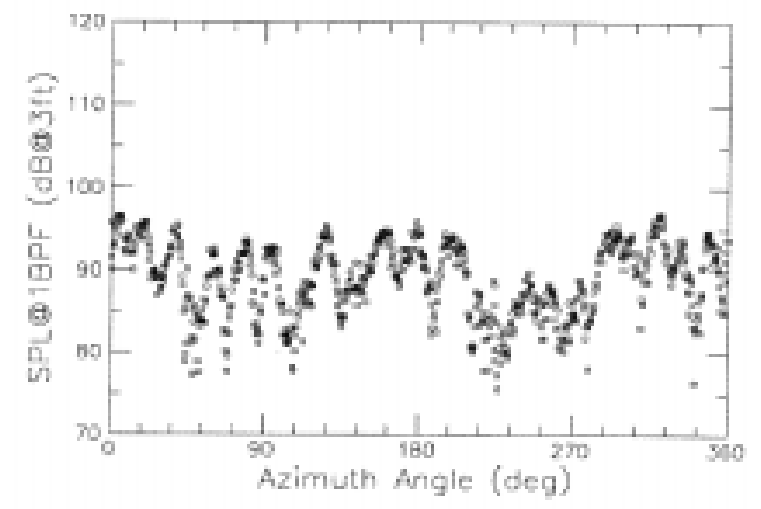

b.) BPF data with 2-second data.

Figure 4. BPF tone level as a function of azimuthal angle at a polar angle of $\psi=90^{\circ}$, a.) with 4-second data, b.) with 2-second data.
Figures 5, 6 and 7 show, respectively, the BPF, $2 \mathrm{BPF}$ and the $3 \mathrm{BPF}$ tones plotted, now for $\psi=45^{\circ}$. The BPF tone patterns are well defined at $\psi=45^{\circ}$. For the harmonic tones, patterns can be seen to be well defined, but there appear to be more patterns superimposed giving the different appearance from the $\mathrm{BPF}$ tone. The sound pressure levels of the BPF tone are high compared to the $2 \mathrm{BPF}$ and $3 \mathrm{BPF}$ tones. In the past, this fan model consistently showed levels of BPF tone that were too high, even without any apparent generation mechanism present. However, in this experiment the presence of the baffle wall enhances this by lowering the levels of the 2BPF tone. The 2BPF tone is an aft dominant tone that was prevented from raising the forward radiated level by the baffle wall. This was observed by comparing the current results with those of the previous experiment on this fan in Reference 6.

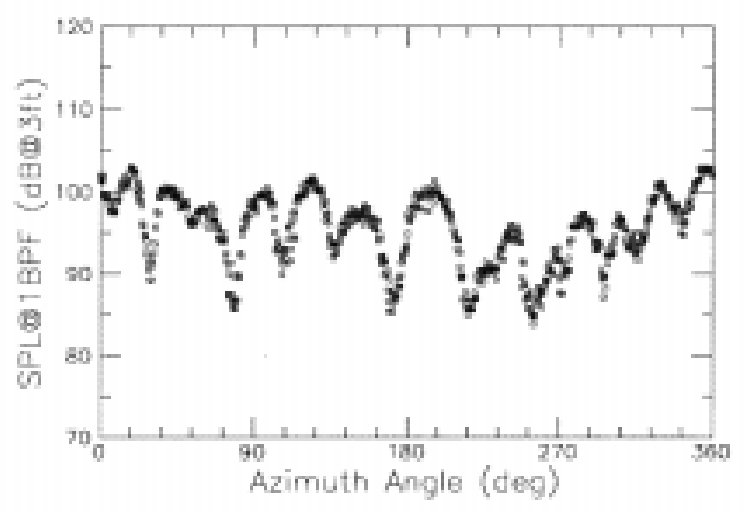

Figure 5. BPF tone level as a function of azimuthal angle at a polar angle of $\psi=45^{\circ}$.

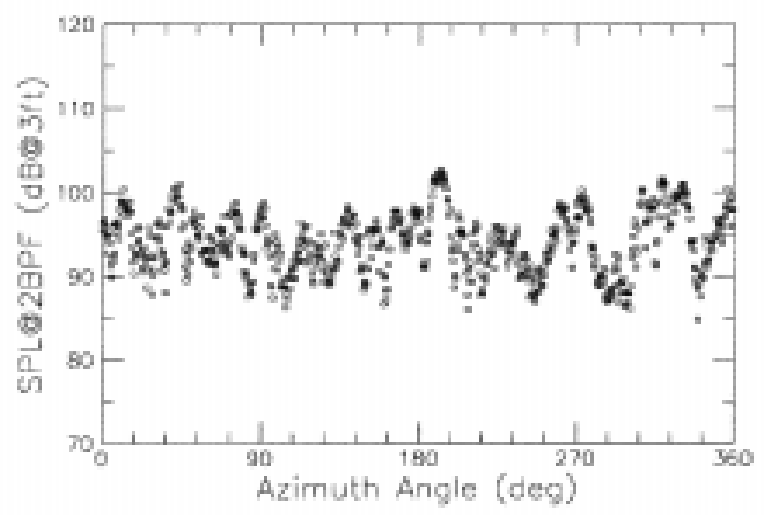

Figure 6. 2BPF tone level as a function of azimuthal angle at a polar angle of $\psi=45^{\circ}$. 


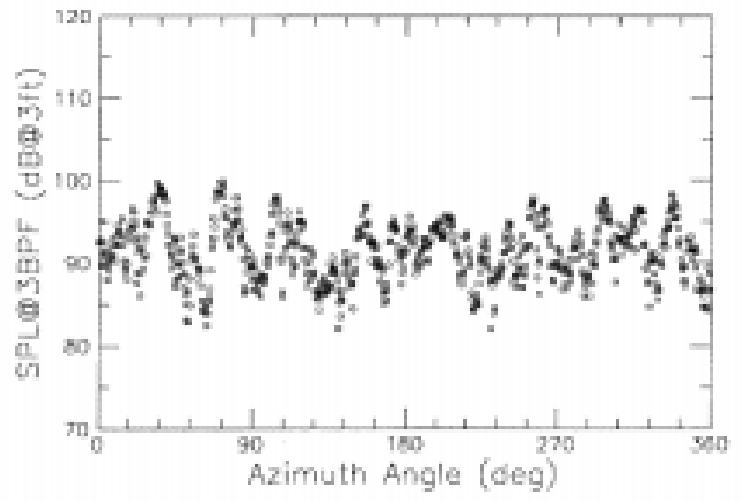

Figure 7. 3BPF tone level as a function of azimuthal angle at a polar angle of $\psi=45^{\circ}$.

Figures 8, 9, and 10 show the BPF, 2BPF, and $3 \mathrm{BPF}$ tones, respectively, plotted as a function of azimuthal angle. These figures are taken at the axial station corresponding to the polar angle of $\psi=$ $20^{\circ}\left(19.98^{\circ}\right.$ actual). The patterns in the BPF are significantly different than at $\psi=45^{\circ}$, Figure 5 . Noticeable differences can also be seen in the $2 \mathrm{BPF}$ and 3BPF tones between $\psi=20^{\circ}$ and $45^{\circ}$.

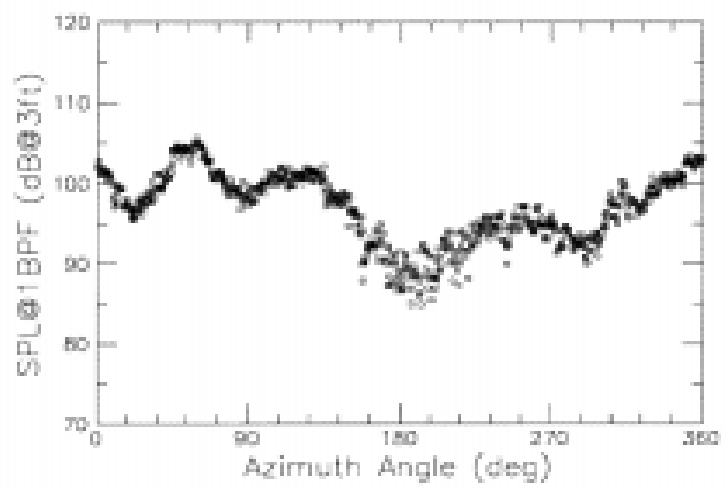

Figure 8. BPF tone level as a function of azimuthal angle at a polar angle of $\psi=20^{\circ}$.

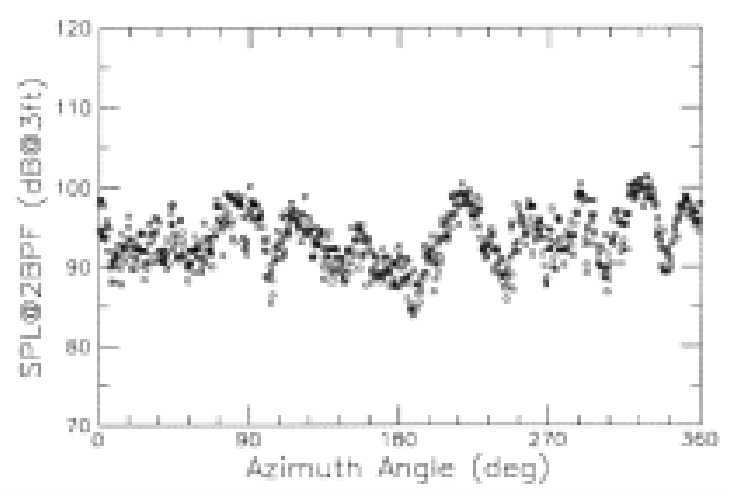

Figure 9. 2BPF tone level as a function of azimuthal angle at a polar angle of $\psi=20^{\circ}$.

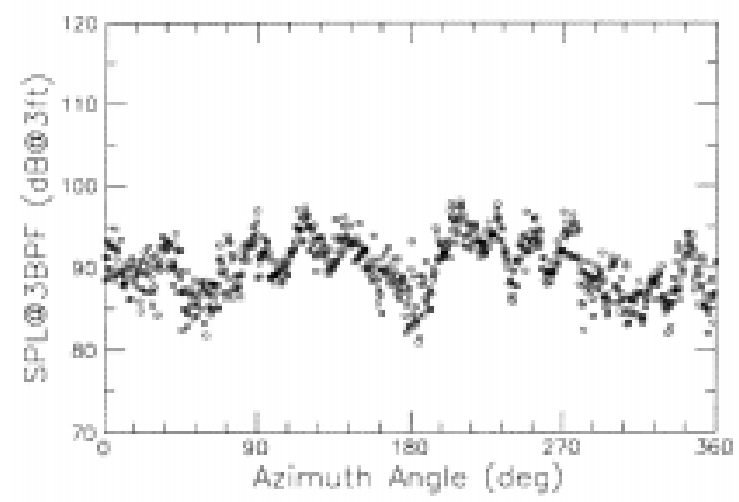

Figure 10. 3BPF tone level as a function of azimuthal angle at a polar angle of $\psi=20^{\circ}$.

The data taken by the circular array at several axial stations have the form of a cylindrical surface around the axis of the fan. When this data is plotted together, 736 points at each of 14 axial stations, and unwrapped to a flat surface the result is a contour plot. Figure 11 is the contour plot for the BPF tone in a gray scale (the full color plot contains much more detail). Because of the repeatability results, Figure 11 represents the full far field pattern of the BPF that is stationary in space.

Figure 12 is the gray scale contour for the $3 \mathrm{BPF}$ tone. The $3 \mathrm{BPF}$ tone was also shown in the previously reported experiment, Figure 12 of Reference 6 . In that experiment, 40 distinct lobes were present in the $3 \mathrm{BPF}$ tone for polar angles from $\psi=110^{\circ}$ to about $75^{\circ}$. Using the mode detection method, under development in Reference 6, the 40 lobes were attributed to the $m=8$ circumferential mode due to rotor-stator interaction. In Figure 12 of this paper those 40 lobes are not clearly present and that is due to the presence of the baffle wall separating aft and forward radiating noise interaction.

\section{Circular Microphone Array for Mode Detection Theory}

The theory of using a circular microphone array parallel to the fan face whose center lies on the fan axis was given by Farassat and Myers ${ }^{10}$. It was shown that the circumferential Fourier transformation of the complex acoustic pressure spectrum at several axial distances from the fan face could give the modal structure of the propagated modes. We give a summary of the results of this reference here for acoustic radiation from a stationary ducted fan inlet.

First, we introduce the following additional symbols used in the development of the method. The geometry of the problem is depicted in Fig. 13.

a: $\quad$ radius of microphone array 


$\begin{array}{ll}B: & \text { number of blades } \\ c: & \text { speed of sound in undistrubed medium } \\ k_{r}: & \text { radial wave number } \\ m: & \text { circumferential model number } \\ n: & \text { radial mode number } \\ r: & \text { radial distance from duct axis } \\ r_{o}: & \text { duct radius } \\ \mathrm{R}: & \text { distance of observer from source point on duct } \\ & \text { inlet or exhaust } \\ R_{o}: & \text { distance of observer from duct center } \\ V: & \text { number of stator vanes or number of } \\ \alpha: & \text { circumferentially periodic disturbances } \\ \Omega: & \text { multiples of BPF } \\ \theta^{\prime}: & \text { angular velocity of the rotor } \\ & \text { array of an individual microphone }\end{array}$

Let $m$ be the circumferential and $n$ the radial mode numbers of a spinning mode. Assume $V$ vanes or periodic circumferential disturbances interacting with $B$ rotor blades. Then, the circumferential mode numbers generated from the interaction are given by

$$
m=\alpha B+q V
$$

where $\alpha=1,2, \ldots$, and $q=0, \pm 1, \pm 2, \ldots$ We write the propagating acoustic pressure at the inlet or exhaust of the duct as follows

$$
p_{S}^{\prime}(r, \theta, t)=e^{i \alpha \beta(\Omega t-\theta)} \sum_{n} \sum_{q} A_{n q} J_{m}\left[k_{r}(m, n) r\right] e^{-i q V \theta}
$$

where $\Omega$ is the shaft frequency, $r$ is the radial distance and $k_{r}$ is the radial wave number of mode $(m, n)$. Note that $m=m(q)$ by Eq. (13). Using Eq. 14 in the Rayleigh formula

$$
4 \pi p^{\prime}(\vec{x}, t)=\frac{k_{a}}{\alpha B \Omega} \frac{\partial}{\partial t} \int_{S}^{\left[p_{S}^{\prime}\right]_{r e t}} \frac{R}{R} d S
$$

where $R=|\vec{x}-\vec{y}|, \vec{y}$ is a point on the duct inlet or exhaust surface $S$ and the subscript ret denotes retarded time.

Using Eq. (14) in Eq. (15) and integrating, we get in the far field:

$$
p^{\prime}\left(a, \theta^{\prime}, x, t\right)=\frac{i}{4 R_{0}} D\left(\theta^{\prime}, \psi\right) e^{i \alpha s\left[\Omega\left(t-R_{0} / c-\theta^{\prime}\right]\right.}
$$

where

$$
D\left(\theta^{\prime}, \psi\right)=\sum_{q} D_{q}(\psi) e^{-i q q \theta^{\prime}}
$$

$$
D_{q}(\psi)=\sum_{n} k_{a}(m, n) C_{m n}(\psi) A_{n q}
$$

and

$$
C_{m n}(\psi)=\int_{o}^{r_{o}} r J_{m}\left[k_{r}(m, n) r\right] J_{m}\left[\frac{\alpha B \Omega r}{c} \sin \psi\right] d r
$$

In Eq. (18), $k_{a}(m, n)$ is the axial mode number of mode $(m, n)$. We note that $D\left(\theta^{\prime}, \psi\right)$ is found from the complex acoustic spectrum for a given multiple of the blade passage frequency. By circumferential Fourier transformation of $D\left(\theta^{\prime}, \psi\right)$, we get $D_{q}(\psi)$. Since $C_{m n}(\psi)$ and $k_{a}(m, n)$ are analytic results and are known for a given $m, n$ and $\psi$, equation (18) can be solved for $A_{n q}$ using acoustic measurements at several distances from the inlet or exhaust of the duct. Equation (18) is a linear equation in terms of the unknowns $\mathrm{A}_{\mathrm{nq}}$. Note that since $m$ is a function of $q, A_{n q}$ is the complex amplitude of the spinning mode $(m, n)$. The method is summarized in the algorithm of Fig. 14.

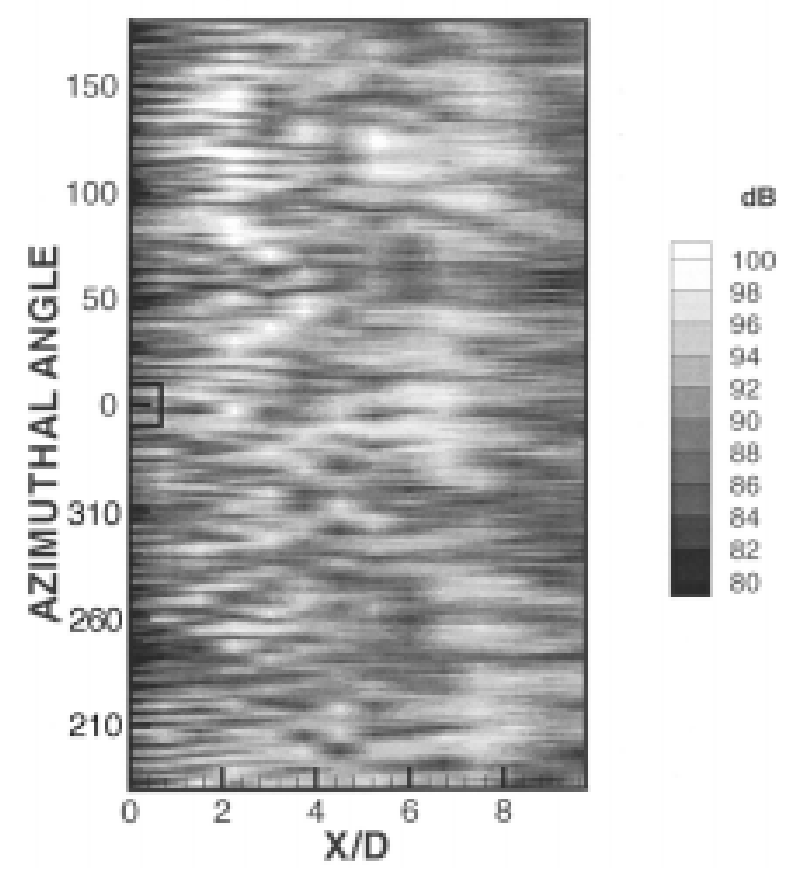

Figure 11. Surface contour plot of the BPF tone level using all azimuthal and polar angles measured in the far field. Outline of fan inlet is shown at the azimuthal angle $\psi=0^{\circ}$. 


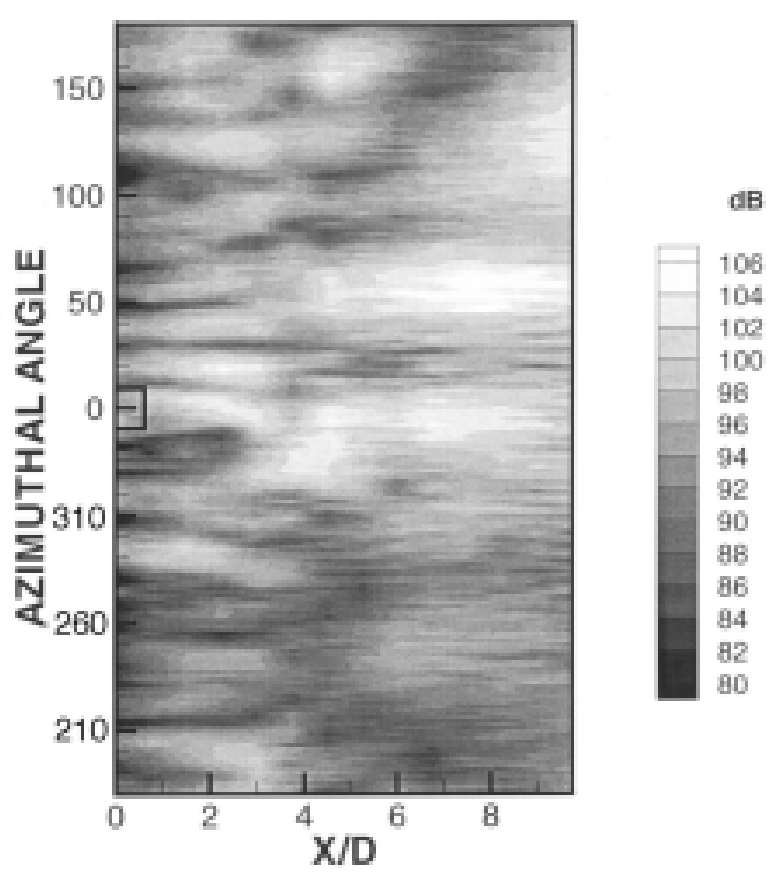

Figure 12. Surface contour plot of the 3BPF tone level using all azimuthal and polar angles measured in the far field. The fan is shown at the azimuthal angle $\psi=0^{\circ}$.

\section{$\underline{\text { Code Development }}$}

A Fortran 77 code was written for the above theory by Dr. J. Masad of Lockheed Martin. Since it was planned to take more axial acoustic data than is necessary for detecting the modes, and because of the possibility of high condition numbers in inverting Eq. (18), Farassat and Myers recommended using singular value decomposition and Tikhonov rigorization ${ }^{11}$ for inverting the linear system. Various tests of the code show that the inversion method is robust and reliable. In the computation of the coefficients of the linear system, only propagating modes are used, i.e., only modes with real axial wave number $k_{a}$.

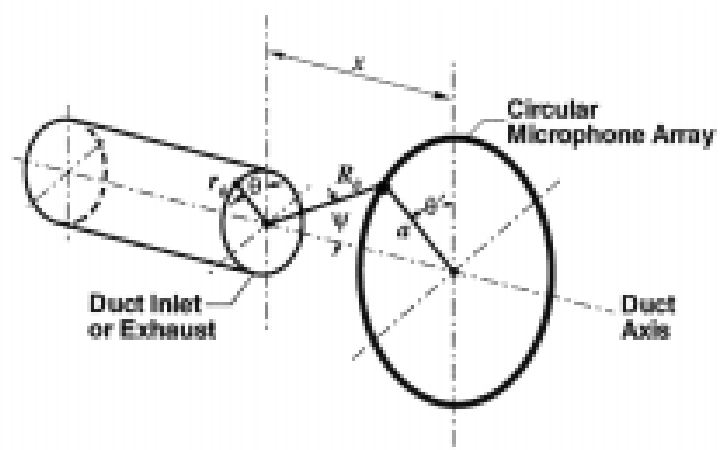

Figure 13. Geometric definitions used in the mode detection method.

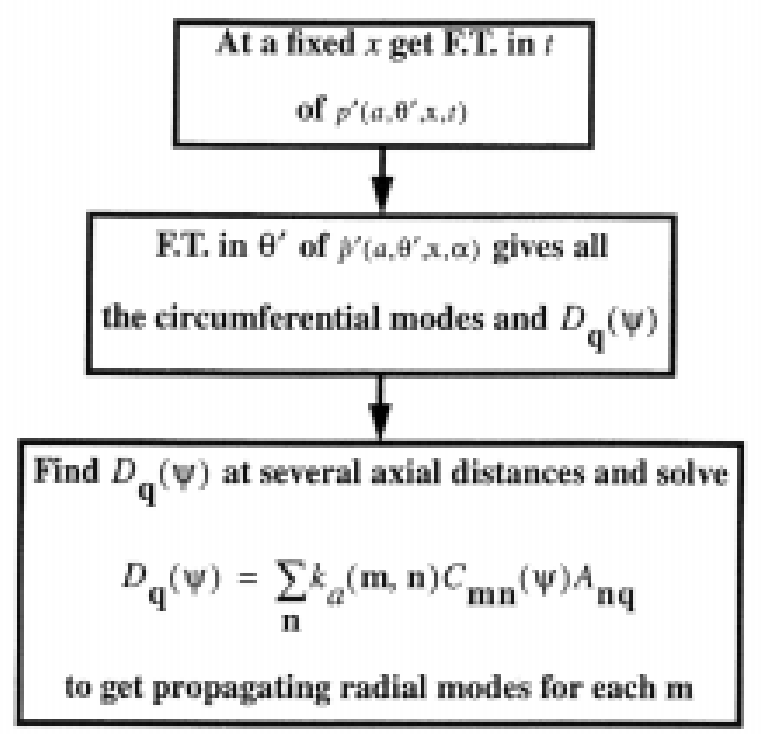

Figure 14. Summary of the algorithm of the mode detection method.

\section{$\underline{\text { Results and Discussion }}$}

The coded mode detection method was used to analyze the azimuthal directivity experiment described earlier. The experimental data used were the full set including all 14 axial stations each with 736 azimuthal data points and all taken with the 4-second data. The average inlet duct Mach number of 0.46 (corresponding to the $100 \%$ speed condition) was also supplied to the coded method.

A value of the periodic circumferential disturbances, $V$, must also be selected in the mode detection method. These circumferential disturbances produce the interactions with the number of blades, $B$. These may come from known sources such as rotorstator interaction or from sources, which have not been identified.

In addition to rotor-stator interaction, other possible known sources are disturbances due to struts, instrument rakes or other wake generating devices installed in the flow duct. A non-uniform rub strip producing fluctuating fan rotor tip loading is another source that has been investigated on this fan previously. ${ }^{4}$ Inflow disturbances or boundary layer nonuniformity are also to be considered, however, their periodicity may not be easily quantified a priori. That is, only the post analysis may be able to assign a value of $V$ to the disturbance. For these reasons, if a value of $V=1$ is chosen in the mode detection method, the result will be all modes detected for all disturbances, identifiable and non-identifiable.

Figure 15 presents the results of the analysis for $V=1$ for the fundamental tone, BPF. For the first radial mode, $n=1$, circumferential modes are detected 
from $m=-12$ to $m=12$. By the third radial mode, $n=$ 3 , circumferential modes are present from $m=-2$ to +2 . In total, about 20 modes are detected that have significant amplitude (greater than $10 \mathrm{~Pa}$ ). It is important to note that with 40 stator vanes the rotorstator interaction at BPF is cut-off. Therefore, the modes present must come from other interactions.

To determine the source of other disturbances more information would be needed. For example, in Figure 15, the $m=10$ mode is one of the modes with significant amplitude. With $B=16, \alpha=1$, and $m=10$, equation (13) would read $10=16+q V$ or $q V=-6$. Given the combinations for $\mathrm{q}$ and $V$, possible values of $V$ would yield $6,3,2$, or 1 . However, there are no known disturbances that may be characterized with these values.

This fan and experiment were designed such that there were no struts or probes in the flow path and great care was taken in making a smooth rub strip and in the elimination of disturbances from other sources such as bolt holes. This would leave other disturbances such as inflow or boundary layer mechanisms that may have periodic disturbances.

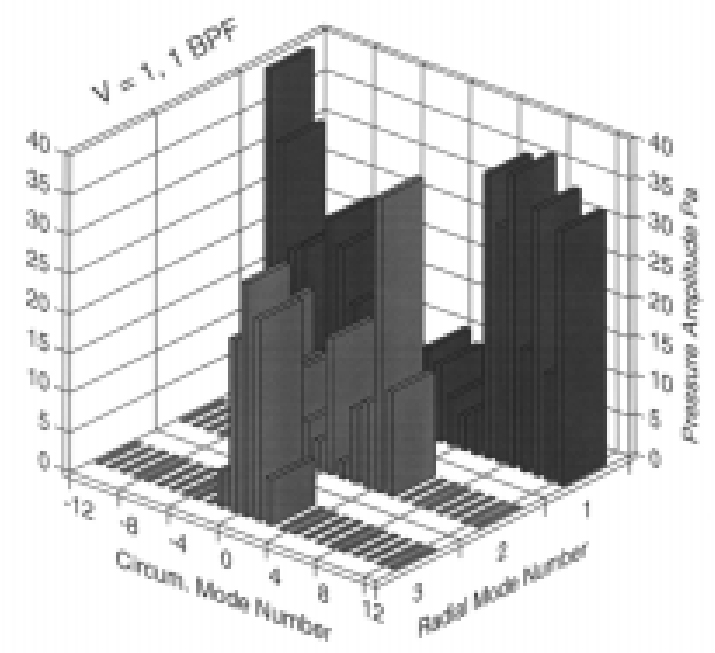

Figure 15. Detected modes at BPF with $V=1$.

All modes which are detected for the $2 \mathrm{BPF}$ mode, with $V=1$, are shown in Figure 16. Up to 8 radial modes are present for $m=-25$ to $m=25$ for a total of many modes detected. As just illustrated, many of these modes come from unidentified sources, however, there are some sources which can be identified.

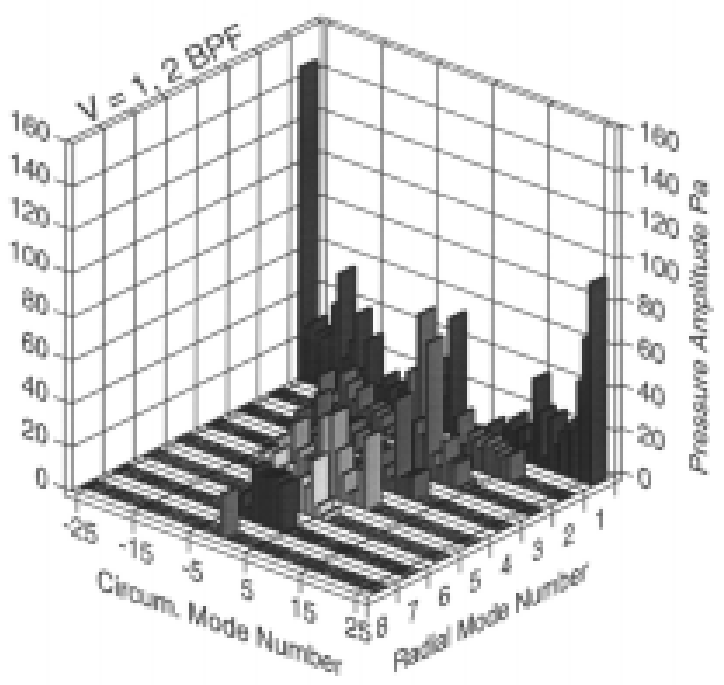

Figure 16. Detected modes at $2 \mathrm{BPF}$ with $V=1$.

The ICD installed on the fan was manufactured with 9 ribs. The wakes off of these ribs, while small, still produce interaction with the rotor. Figure 17 shows the modes detected from $V=9$ at BPF with the significant modes being $m=-11$ and 7 . Both of these modes are included in the plot showing all modes detected at BPF (Figure 15). The ICD ribs produce many more modes at $2 \mathrm{BPF}$ as seen in Figure 18. Again, all these modes are included in Figure 16 but now being identified as originating from the ICD rib-rotor interaction. Significant interaction and mode propagation are also attributed to the ICD ribs at $3 \mathrm{BPF}$ as seen in Figure 19.

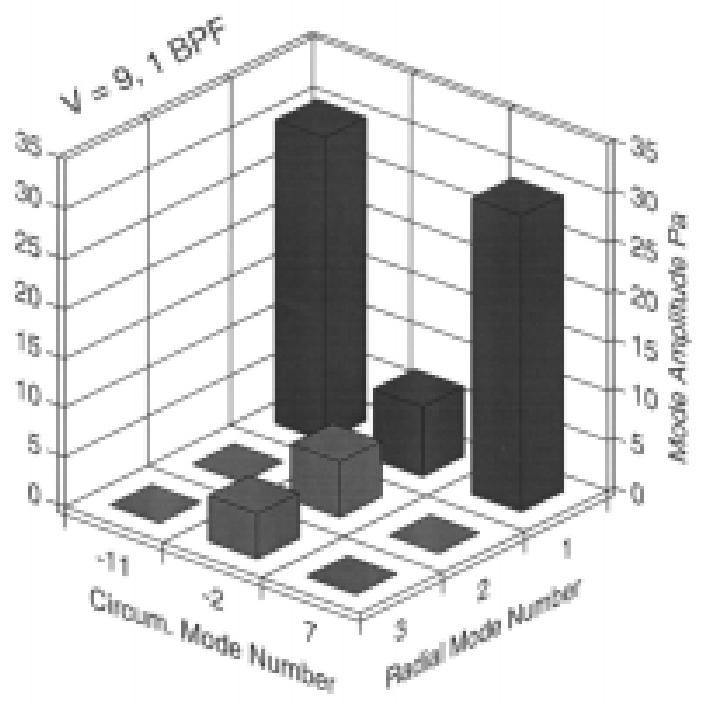

Figure 17. Detected modes at BPF with $V=9$ from ICD support ribs. 


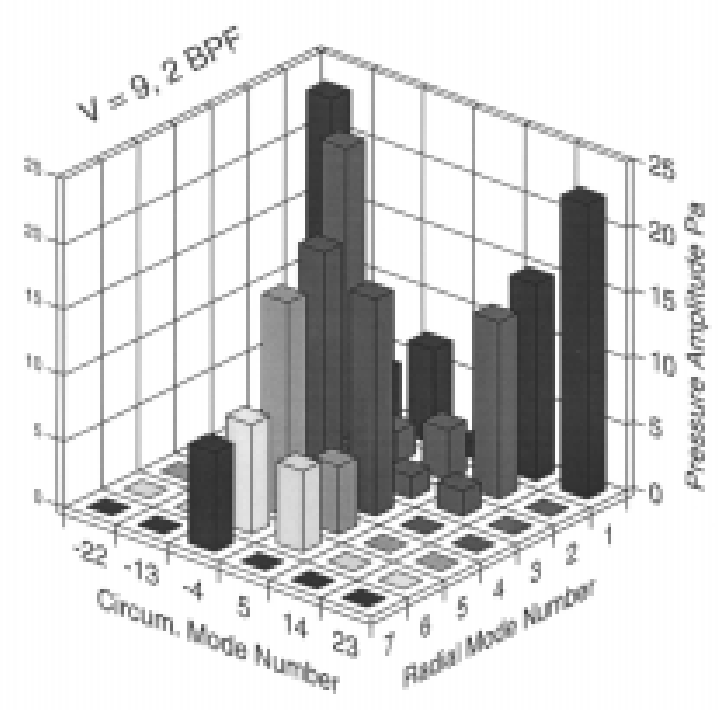

Figure 18. Detected modes at 2 BPF with $V=9$ from ICD support ribs.

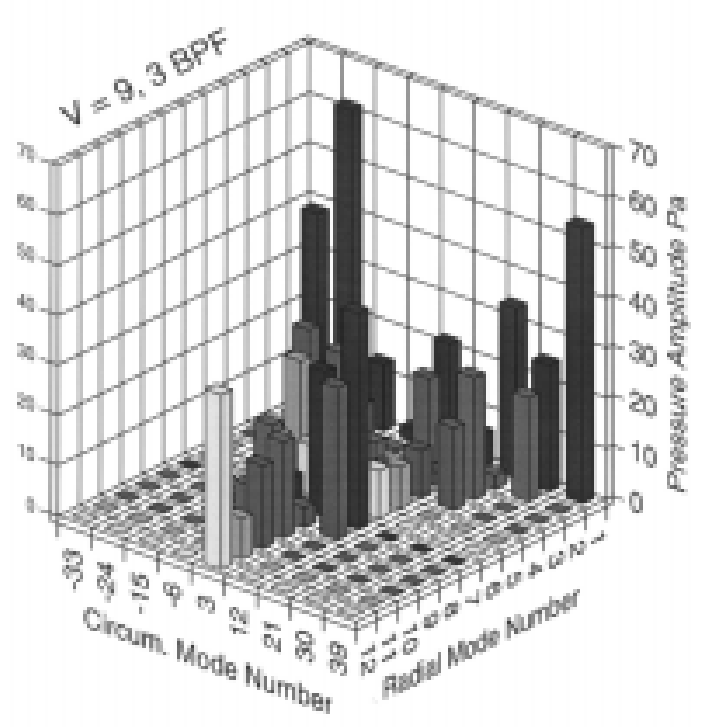

Figure 19. Detected modes at 3BPF with $V=9$ from ICD support ribs.

The other clearly identified interaction source is the rotor-stator interaction with $V=40$. At 2BPF, Figure 20 identifies 5 radial orders for the $m=-8$ mode. These modes were also identified by the rotating rake method of Reference 12 on a fan of exactly the same geometry (but a larger scale at a diameter of 17.25 inches and of different detailed construction).

At 3BPF, the mode detection method identifies 5 significant radial modes for $\mathrm{m}=8$ and one radial mode at $\mathrm{m}=-32$ ( see Figure 21 ). Comparison is not possible since the rotating rake method of Reference 12 was unable to report results at the $3 \mathrm{BPF}$.

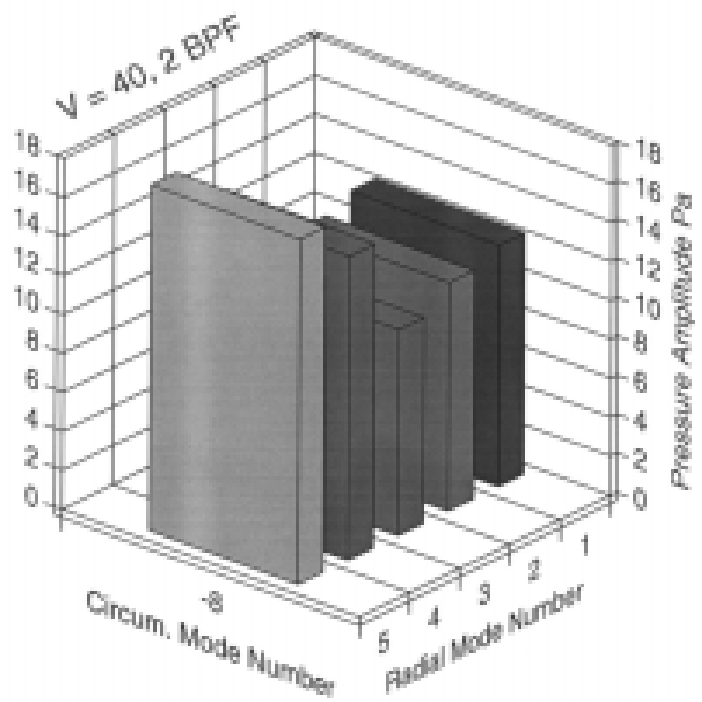

Figure 20. Detected modes at $2 \mathrm{BPF}$ with $V=40$ from stator vanes.

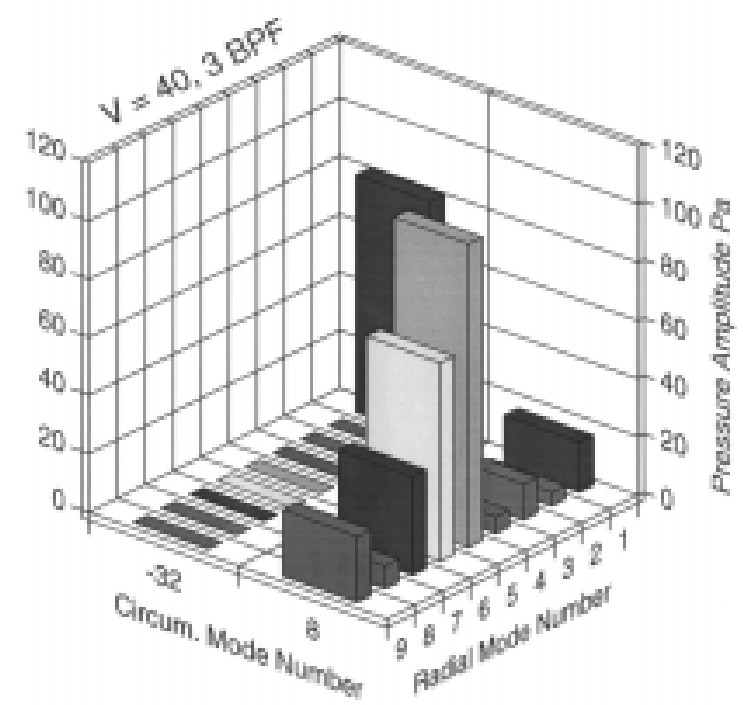

Figure 21. Detected modes at $3 \mathrm{BPF}$ with $V=40$ from stator vanes.

\section{$\underline{\text { Conclusions }}$}

Very high resolution measurements of the far acoustic field of the NASA Langley 12 inch fan have been reported. This mapping of the far field yielded very detailed information about the radiated directivity especially in the azimuthal direction. The azimuthal directivity is significant with variation of $10-15 \mathrm{~dB}$ in the blade passage frequency tones.

In this case, care was taken to minimize the possible sources of interaction with the rotor field. There were no physical disturbances to the flow field other than the stator vanes and the structure of the 
inflow control device. Even so, the azimuthal directivity was significant. Therefore, the general assumption of axisymmetry in the far field of a fan should be examined and validated in each application.

The detailed measurement of the far field was also used to identify the radiated modes that produced the directivity in the far field. For the first time, a method of far field mode detection was used to detect both circumferential and radial modes in the far field.

This method correctly identified the modes that were attributable to the known rotor-stator and rotor-ICD rib interactions. In addition, many other modes were detected without identifiable interaction sources which remains a subject of research.

Further validation of this far field mode detection method also remains including a sensitivity analysis and minimization of the number of measurements needed in the far field. With further development, the far field method of mode detection will present a viable method compared to the previously developed methods of the in duct rotating microphone and the in duct wall microphone array.

Each of the three methods has advantages that will determine the best method for a given application. A significant advantage of the far field method is the mechanical simplicity of the circular array and that the array is independent of the fan or engine tested. Also, the circular array could be used in the inlet and exhaust directions with equal ease without any interference with the flow through the engine. These represent significant advantages for the far field method of mode detection.

\section{Acknowledgement}

R.H. Thomas gratefully acknowledges the support of this work by the Aeroacoustics Branch of NASA Langley Research Center.

\section{$\underline{\text { References }}$}

1. Block, P.J.W., "Installation Noise Measurements of Model SR and CR Propellers," NASA TM-85790, 1984.

2. Tam, C.K.W., Salikuddin, M., and Hanson, D.B., "Acoustic Interference of Counter-Rotation Propellers," Journal of Sound and Vibration, Vol. 124, No. 2, 1988, pp. 357-366.

3. Falarski, M.D. and Moore, M.T., "Acoustic Characteristics of Two Hybrid Inlets at Forward Speeds," Journal of Aircraft, Vol. 17, No. 2, 1980, pp. 106-111.
4. Thomas, R.H., Gerhold, C.H., Farassat, F., Santa Maria, O.L., Nuckolls, W.E., and DeVilbiss, D.W., "Far Field Noise of the 12 Inch Advanced Ducted Propeller Simulator," AIAA Paper No. 95-0722, $33^{\text {rd }}$ Aerospace Sciences Meeting, Reno, NV, January, 1995.

5. Ganz, U., "Multi-Modal Directivities of Fan Tone Noise," NOISE-CON 96, Bellevue, WA, Oct. 1996.

6. Thomas, R.H., Farassat, F., Clark, L.R., and Gerhold, C.H., "Azimuthal Patterns of the Radiated Sound Field from a Turbofan Model," AIAA/CEAS Paper No. 97-1588, $3^{\text {rd }}$ AIAA/CEAS Aeroacoustics Conference, Atlanta, GA, May, 1997.

7. Hodges, R.M., Gerhold, C.H., Balster, D. and Thomas, R.H., "Acoustic Testing of Very High Bypass Ratio Turbofans Using Turbine Powered Scale Models," AIAA Paper No. 94-2552, presented at the $18^{\text {th }}$ Aerospace Ground Testing Conference, June 20-23, 1994.

8. Homyak, L., McArdle, J.G., and Heidelberg, L.J., "A Compact Inflow Control Device for Simulating Flight Fan Noise," AIAA Paper No. 83-0680, April, 1983.

9. Bendat, J.S. and Piersol, A.G., Random Data: Analysis and Measurement Procedures, WileyInterscience, New York, 1986.

10. Farassat, F., and Myers, M.K., "A Study of Wave Propagation in a Duct and Mode Radiation," AIAA 96-1677, 1996.

11. Tikhonov, A. V. and Arsenin, V. Y., Solution of Ill-posed Problems, 1977, Winston and Sons.

12. Heidelberg, L. J., Hall, D. G., "Inlet Acoustic Mode Measurements Using a Continuously Rotating Rake," Journal of Aircraft, Vol. 32, No. 4, July-August 1995, pp. 761-767. 